\title{
Evaluation of Axial Length Measurement Using Enhanced Retina Visualization Mode of the Swept-Source Optical Coherence Tomography Biometer in Dense Cataract
}

\author{
Akeno Tamaoki $^{a}$ Takashi Kojima ${ }^{a, b}$ Asato Hasegawa ${ }^{a}$ Mana Yamamoto ${ }^{a, c}$

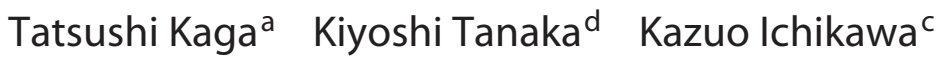 \\ aDepartment of Ophthalmology, Japan Community Healthcare Organization Chukyo Hospital, Nagoya, Japan; \\ bepartment of Ophthalmology, Keio University School of Medicine, Tokyo, Japan; 'Chukyo Eye Clinic, Nagoya, \\ Japan; ${ }^{d}$ Department of Mathematics and System Development, Shinshu University Interdisciplinary Graduate \\ School of Science and Technology, Nagano, Japan
}

\section{Keywords}

Axial length · Cataract surgery · Enhanced retina

visualization · Intraocular lens power calculation

\begin{abstract}
Introduction: It has been reported that even using the swept-source (SS) optical coherence biometer, it is challenging to measure the axial length $(A L)$ in cases with advanced cataracts. The enhanced retina visualization (ERV) mode, which is equipped with OCTB1 (ARGOS), shifts the peak of measurement sensitivity to the retinal side so that the $A L$ can be measured even if the light energy is attenuated. The aim of the present study was to evaluate the accuracy and efficacy of the ERV mode in measuring the AL of dense cataracts. Methods: This was a single-center retrospective observational case series conducted in Japan. We included 213 eyes of 213 consecutive patients with advanced cataracts who underwent preoperative evaluation. The AL was measured before and after surgery using two SS optical coherence tomography biometers (OCTB1 and OCTB2; IOLMaster 700). Cases in which OCTB1 the standard mode failed to measure AL, OCTB1 with the ERV mode was used instead. Primary outcome measures were the acquisition rate and the $A L$ mea-
\end{abstract}

surement accuracy using the ERV mode. The $x^{2}$ test, the Kruskal-Wallis test, and the Wilcoxon signed-rank test were used to compare the acquisition rate and differences between pre- and postoperative AL values, respectively. In the ERV subgroup, Bland-Altman plots were used to compare the pre- and postoperative AL values measured using OCTB1ERV mode. A $p$-value of less than $5 \%$ was considered statistically significant. Results: The AL acquisition rate was not significantly different between OCTB1 with the standard mode and OCTB2. The AL of 65 eyes (30.5\%) could not be measured using OCTB1 with the standard mode. Conversely, the AL of 51 of these eyes (78.5\%) was successfully measured using OCTB1 with the ERV mode. In these 51 eyes, a difference of $\leq 0.2 \mathrm{~mm}$ and of $\leq 0.1 \mathrm{~mm}$ between pre- and postoperative $\mathrm{AL}$ measurements was observed in 40 (78.4\%) and 30 eyes (58.8\%), respectively. The Bland-Altman plot found no systematic error between pre- and postoperative $A L$ values measured using the ERV mode. Conclusion: In patients with dense cataracts, AL measurement using the standard mode of an SS-OCT biometer is challenging. Furthermore, the ERV mode could be promising for AL measurement in such cases.

(C) 2021 The Author(s)

Published by S. Karger AG, Basel
(C) 2021 The Author(s)

Published by S. Karger AG, Basel

This is an Open Access article licensed under the Creative Commons Attribution-NonCommercial-4.0 International License (CC BY-NC) (http://www.karger.com/Services/OpenAccessLicense), applicable to the online version of the article only. Usage and distribution for commercial purposes requires written permission.
Correspondence to:

Takashi Kojima, tkojkoj@mac.com 


\section{Introduction}

Reduction of the predictive refractive error after cataract surgery is important to improve patient satisfaction [1]. Accurate biometric measurements are essential to achieve that goal. Among the biometric measurements, the axial length $(\mathrm{AL})$ is one of the important factors that influence the predictive refractive error [2-4].

The development of partial coherence interferometry (PCI) has improved the accuracy of AL measurement, which is higher than that of ultrasound (US) biometry. The main reason for this accuracy is that PCI does not involve contact with eyes and that it is less dependent on the skill of the examiner; moreover, it is associated with a higher directivity and better reproducibility than US biometry [5]. Therefore, PCI has replaced US biometry. However, it has been reported that AL cannot be measured in $6.4-37.8 \%$ of cases because of various reasons such as poor eye fixation, dense cataract, or corneal abnormalities [6-14]. Among them, the main cause has been reported to be media opacity in the visual axis in cases such as those involving posterior subcapsular cataracts $[11,15]$. The newly introduced swept-source optical coherence tomography (SS-OCT) device succeeded in attenuating Rayleigh scattering [16] that occurs because of cataract by using long-wavelength light as a light source, and it became possible to measure the AL in patients with posterior subcapsular cataracts that cannot be measured by PCI. However, even by using the SS-OCT device, it has been reported that the AL cannot be measured in $0.6-7.4 \%$ of cases $[14,17,18]$. Therefore, measurement by US biometer is still necessary for advanced cataract.

Although US biometry is effective in measuring AL especially in patients with advanced cataract, in whom an optical biometer cannot measure AL, it also has some disadvantages, such as the following: (1) variations in the measured values owing to contact with the cornea, (2) the immersion method requires higher technical expertise and is associated with a poorer reproducibility than the optical biometer, (3) the visual axis cannot be captured, and (4) the anterior and posterior corneal planes are not separated even by the segmented method, and the measurement of accuracy varies depending on the examiner's expertise [19].

The novel enhanced retina visualization (ERV) mode is a unique function to the SS-OCT biometer (OCTB1). In principle, the sensitivity of OCT decreases with depth. Especially in the presence of a dense cataract, detection of the retinal pigment epithelium layer and measurement of

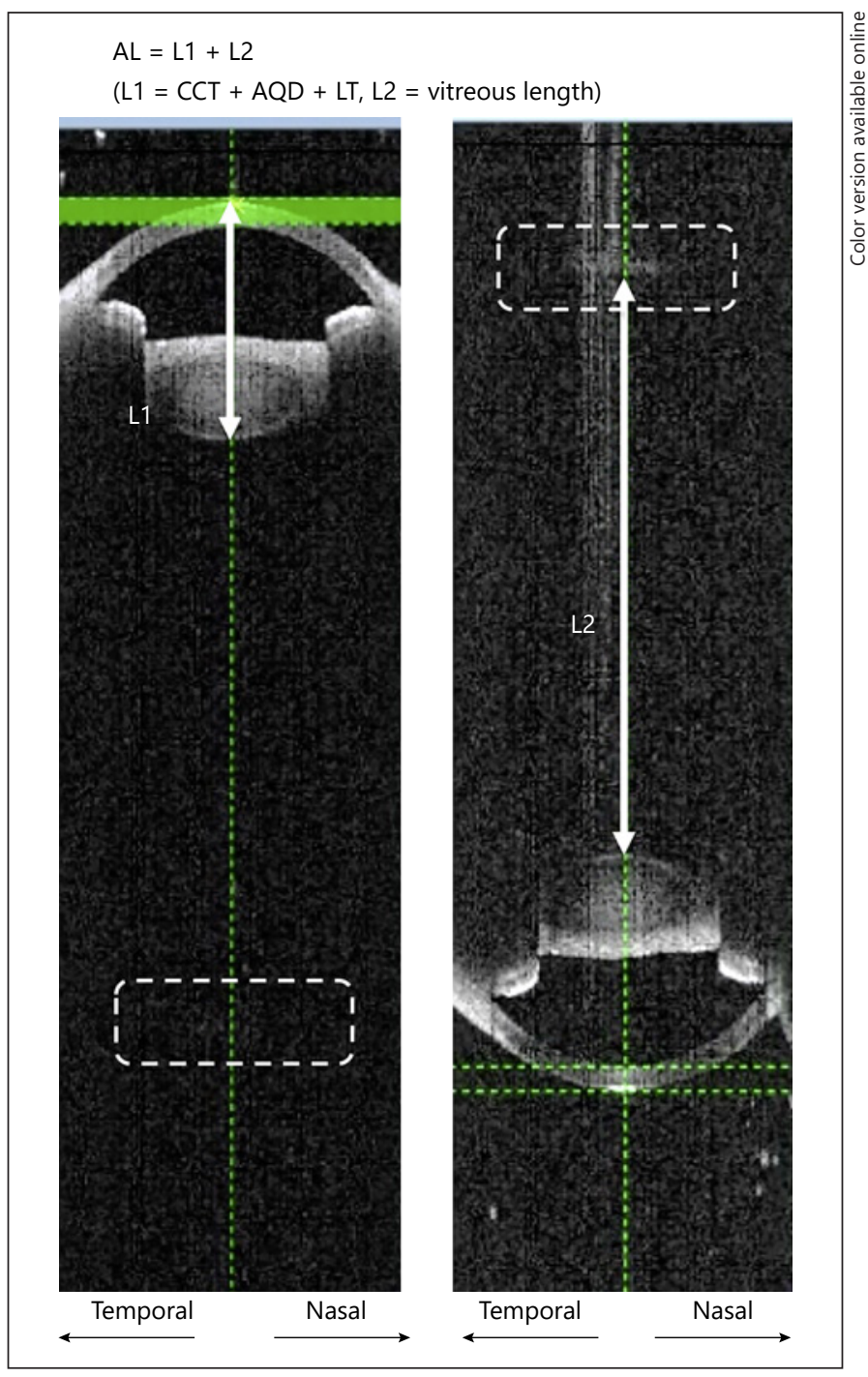

Fig. 1. Principle of AL measurement in OCTB1-ERV mode. In advanced cataract, the optical biometer cannot detect the retina; therefore, the anterior segment (L1; CCT, AQD, and LT) is measured using the standard mode (a), and the vitreous length (L2) is measured using the ERV mode (b). Subsequently, the AL is calculated by the sum of L1 and L2. AL, axial length; CCT, central corneal thickness; AQD, aqueous depth; LT, lens thickness; ERV, enhanced retina visualization; OCTB1-ERV, optical coherence tomography biometer with the ERV mode.

the vitreous length cannot be performed because of the attenuation of light energy. In the ERV mode, the optical path length is measured by minimizing the effect of attenuation and by changing the OCT sensitive position to the retinal side by using the same principle as that used by enhanced depth imaging in choroidal imaging [20]. By combining this optical path length with the anterior seg- 
Table 1. Demographic patient information and preoperative measurement values

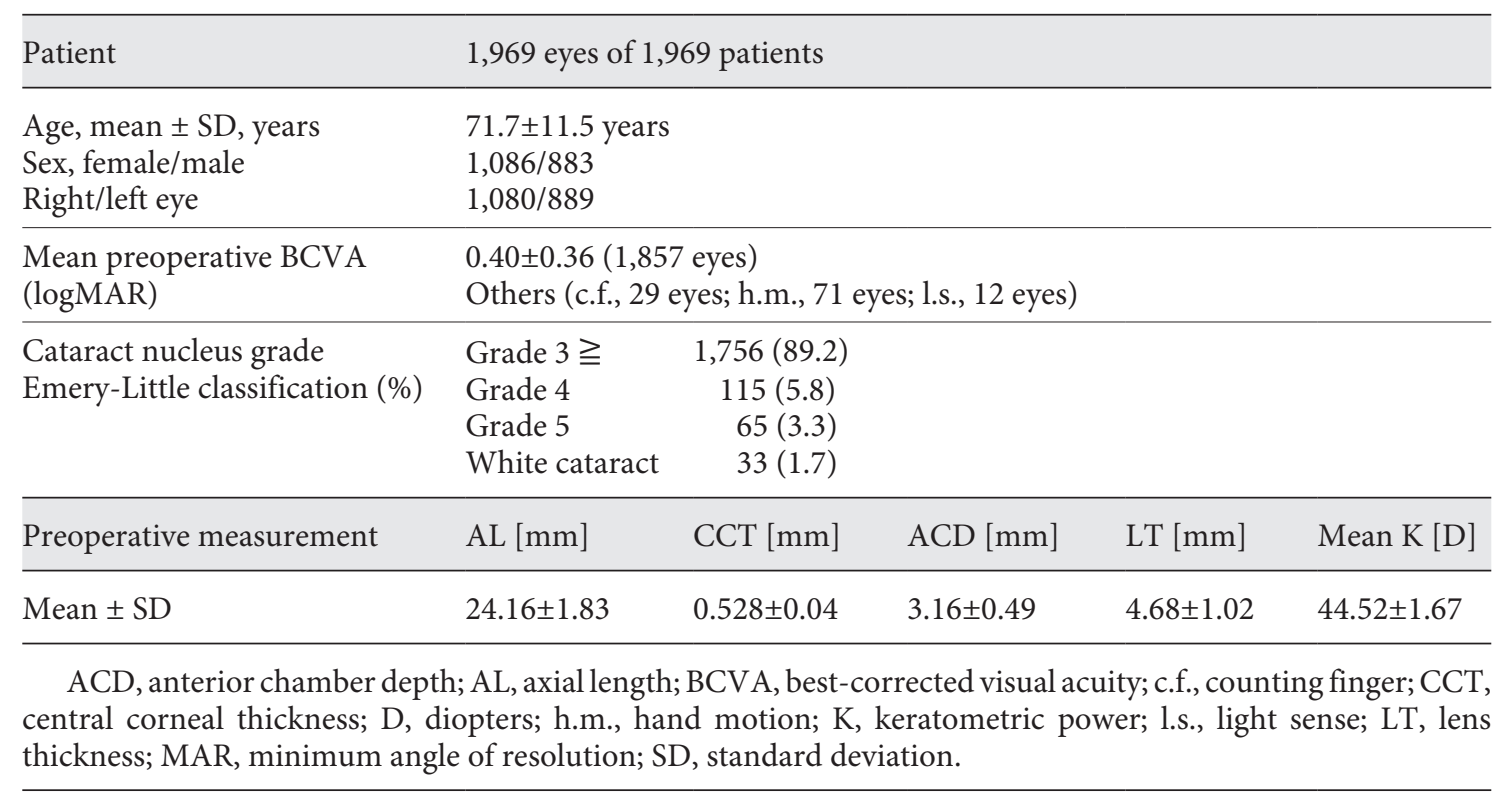

ment information up to the posterior surface of the crystalline lens, measured using the standard mode, the AL in ERV mode is calculated (Fig. 1). Therefore, when the AL is measured by the standard mode, it is not necessary to use the ERV mode.

We have previously conducted a study comparing the gold standard SS-OCT biometer (OCTB2) with OCTB1 [18]. In all cases, OCTB1 (97.6\%) and OCTB2 (92.6\%) showed similarly high measurement rates. However, in advanced cataract, the measurement rate of OCTB1 (89.9\%) was clinically significantly higher than that of OCTB2 (63.6\%). We hypothesized that this difference in measurement rates in patients with advanced cataracts is related to the fact that OCTB1 is equipped with the ERV mode. In this study, to verify this hypothesis, we examined the measurement rate and accuracy of the ERV mode of OCTB1 in measuring AL in patients with advanced cataracts.

\section{Materials and Methods}

The protocol of this study was approved by the Institutional Review Board at Japan Community Healthcare Organization (JCHO) Chukyo Hospital (\#2017026), and the study procedures conformed to the ethical principles for research involving human participants as outlined in the Declaration of Helsinki. As this study was performed retrospectively, the use of an opt-out method was approved by the institutional review board instead of obtaining a written informed consent.

Axial Length Measurement Using

Enhanced Retina Visualization Mode
We retrospectively pre-screened 1,969 consecutive patients who underwent preoperative examination for cataract surgery from July 2016 to March 2019 at the JCHO Chukyo Hospital (Table 1). We subsequently selected patients with white cataract or cataract showing a nuclear hardness grade of $\geq 4$. The nuclear hardness grade was determined according to the Emery-Little classification system [21]. With regard to patients who had undergone examination of both eyes, eyes with a higher cataract grade were selected; if both eyes had the same cataract grade, one of them was randomly selected. As a method for random selection, a random number table created in Excel (Microsoft, Redmond, WA) was used. Furthermore, among the mentioned patients, those in whom AL had been measured both before and after surgery were selected. Finally, 213 eyes of 213 patients were registered in the study. Table 2 shows the demographic information of the patients in eyes with nuclear hardness grade 4 or higher. There were 115 (54.0\%), $65(30.5 \%)$, and 33 patients (15.5\%) with grade 4 , grade 5 , and white cataracts, respectively, among the included patients.

We routinely measure ocular biometry using SS-OCT biometer devices OCTB1 (ARGOS UI version 1.7.0, Movu/Santec, Komaki, Japan) and OCTB2 (IOLMaster700 version 1.80.10, Carl Zeiss Meditec AG, Jena, Germany) to ensure the accuracy of the measurements. We measured the AL several times (at least twice) when we could not measure it from each device. If the AL could not be measured using OCTB1 with the standard mode (OCTB1-standard mode), it was measured using OCTB1 equipped with the ERV mode (OCTB1-ERV mode) and the contact method of US biometer (US-4000 NIDEK, Gamagori, Japan). In addition, we remeasured AL 3 months after the cataract surgery.

\section{AL Measurement Using ERV Mode}

The ERV mode in the OCTB1 was developed to measure AL, when the standard mode failed the measurement. If the AL could not be measured with the OCTB1-standard mode, the ERV mode was selected by examiners. Patients who required the ERV mode 
Table 2. Demographic patient information and preoperative measurement values in eyes with nuclear hardness grade 4 or higher

\begin{tabular}{|c|c|c|c|c|c|}
\hline Patient & \multicolumn{5}{|c|}{213 eyes of 213 patients } \\
\hline Age, mean $\pm S D$, years & \multicolumn{5}{|l|}{$71.9 \pm 14.8$ years } \\
\hline Sex, female/male & \multicolumn{5}{|l|}{$116 / 97$} \\
\hline Right/left eye & \multicolumn{5}{|l|}{$106 / 107$} \\
\hline Mean preoperative & \multirow{2}{*}{\multicolumn{5}{|c|}{$\begin{array}{l}1.04 \pm 0.61 \text { ( } 131 \text { eyes) } \\
\text { Others (c.f., } 20 \text { eyes; h.m., } 54 \text { eyes; 1.s., } 8 \text { eyes) }\end{array}$}} \\
\hline BCVA (logMAR) & & & & & \\
\hline \multirow{3}{*}{$\begin{array}{l}\text { Cataract nucleus grade } \\
\text { Emery-Little classification (\%) }\end{array}$} & Grade 4 & $115(54.0)$ & & & \\
\hline & Grade 5 & $65(30.5)$ & & & \\
\hline & White cataract & $33(15.5)$ & & & \\
\hline Preoperative measurement & $\mathrm{AL}[\mathrm{mm}]$ & $\mathrm{CCT}[\mathrm{mm}]$ & $\mathrm{ACD}[\mathrm{mm}]$ & $\mathrm{LT}[\mathrm{mm}]$ & Mean K [D] \\
\hline Mean \pm SD & $24.51 \pm 2.27$ & $0.536 \pm 0.03$ & $3.21 \pm 0.60$ & $4.60 \pm 0.64$ & $44.36 \pm 1.62$ \\
\hline
\end{tabular}

ACD, anterior chamber depth; AL, axial length; BCVA, best-corrected visual acuity; c.f., counting finger; CCT, central corneal thickness; D, diopters; h.m., hand motion; K, keratometric power; l.s., light sense; LT, lens thickness; MAR, minimum angle of resolution; SD: standard deviation.

Table 3. Demographic patient information and preoperative measurement values in the ERV subgroup

\begin{tabular}{|c|c|c|c|c|c|}
\hline Patient & \multicolumn{5}{|c|}{51 eyes of 51 patients } \\
\hline Age, mean $\pm S D$, years & \multicolumn{5}{|l|}{$64.6 \pm 19.5$ years } \\
\hline Sex, female/male & \multicolumn{5}{|l|}{$30 / 21$} \\
\hline Right/left eye & \multicolumn{5}{|l|}{$28 / 23$} \\
\hline Mean preoperative & \multirow{2}{*}{\multicolumn{5}{|c|}{$\begin{array}{l}2.0 \pm 0.0 \text { ( } 5 \text { eyes) } \\
\text { Others (c.f., } 12 \text { eyes; h.m., } 29 \text { eyes; l.s., } 5 \text { eyes) }\end{array}$}} \\
\hline BCVA (logMAR) & & & & & \\
\hline \multirow{3}{*}{$\begin{array}{l}\text { Cataract nucleus grade } \\
\text { Emery-Little classification (\%) }\end{array}$} & Grade 4 & $8(15.7)$ & & & \\
\hline & Grade 5 & $26(51.0)$ & & & \\
\hline & White cataract & $17(33.3)$ & & & \\
\hline Preoperative measurement & $\mathrm{AL}[\mathrm{mm}]$ & $\mathrm{CCT}[\mathrm{mm}]$ & $\mathrm{ACD}[\mathrm{mm}]$ & $\mathrm{LT}[\mathrm{mm}]$ & Mean K [D] \\
\hline Mean \pm SD & $24.60 \pm 2.14$ & $0.540 \pm 0.04$ & $3.08 \pm 0.71$ & $4.69 \pm 0.80$ & $44.03 \pm 1.60$ \\
\hline $\begin{array}{l}\text { ACD, anterior chamber dept } \\
\text { central corneal thickness; D, } \\
\text { thickness; MAR, minimum ang }\end{array}$ & $\begin{array}{l}\text { L, axial length; } B \\
\text { ters; h.m., hand } \\
\text { f resolution; SD, }\end{array}$ & $\begin{array}{l}\text { A, best-corr } \\
\text { tion; K, ke } \\
\text { adard deviat }\end{array}$ & $\begin{array}{l}\text { d visual acuit } \\
\text { metric powe }\end{array}$ & $\begin{array}{l}\text {; c.f., counti } \\
\text {; l.s., light }\end{array}$ & $\begin{array}{l}\text { g finger; CCT, } \\
\text { ense; LT, lens }\end{array}$ \\
\hline
\end{tabular}

often had a poor vision and could not see the fixation target well. For this reason, the examiners guided the patients to fixate on the target by indicating direction of the target while looking at the whole eye OCT image. All examinations were performed by trained orthoptists.

\section{Outcome Measures}

1. Acquisition rate of the AL measurement

In all cases of nuclear hardness grade 4 or higher (213 eyes), we evaluated the acquisition rate of the AL measurement using two SS-OCT biometers. Subsequently, we evaluated the acquisition rate of the AL measurement using the ERV mode in cases where OCTB1 with the standard mode could not be used.

2. Measurement of the accuracy of AL measurement using the ERV mode

To assess the accuracy of preoperative AL measurements using the OCTB1-ERV mode, pre- and postoperative AL measures were compared in patients in whom AL was successfully measurable with the OCTB1-ERV mode (ERV subgroup). Table 3 shows the patients' demographic information and preoperative measurement values in the ERV subgroup. Among them, there were 8 $(15.7 \%)$ with grade 4 cataracts and $26(51.0 \%)$ with grade 5 cata- 
Fig. 2. Comparison of AL measurement between OCTB1 and OCTB2. Total AL acquisition rate of OCTB1 using the standard mode and ERV mode was significantly higher than that of OCTB2 (a, $p<0.001)$. The accuracy of the preoperative AL measurement using the ERV mode was evaluated by comparing it with the postoperative AL. A difference of $\leq 0.2 \mathrm{~mm}$ was observed in $78.4 \%$ of the cases; however, $9.8 \%$ of the cases showed a difference of $>0.3 \mathrm{~mm}$ (b). $\mathrm{AL}$, axial length; ERV, enhanced retina visualization; OCTB1, optical coherence tomography biometer using the ARGOS device; OCTB2, optical coherence tomography biometer using the IOLMaster700 device.

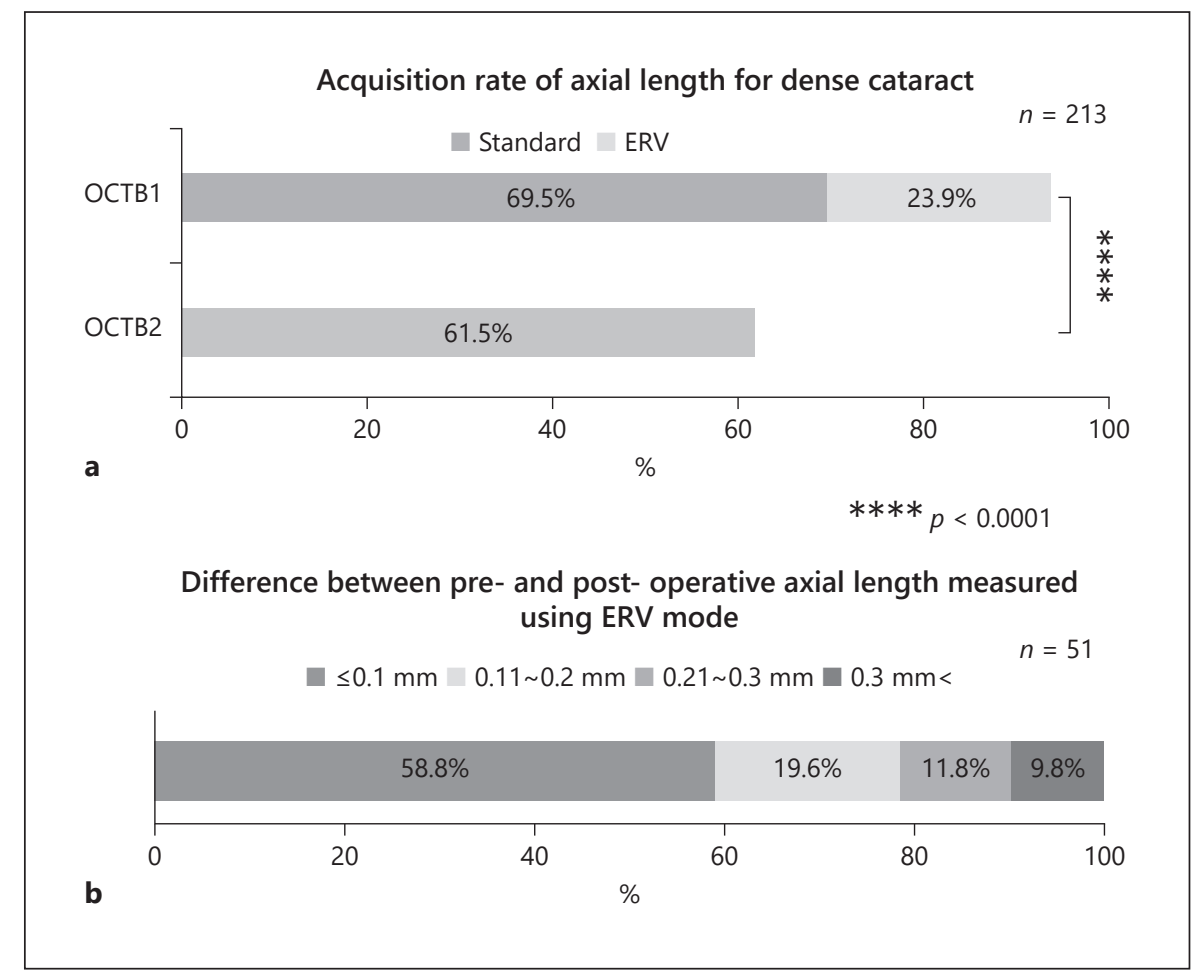

racts according to Emery-Little classification. Additionally, $17 \mathrm{pa}$ tients $(33.3 \%)$ presented white cataracts. OCTB1-standard mode was used for postoperative AL measurement.

\section{Statistical Analyses}

The $\chi^{2}$ test was used to compare the acquisition rate between the two models. The Kruskal-Wallis test was used to compare differences between pre- and postoperative AL values in different patient groups. The Wilcoxon signed-rank test was used to compare differences in pre- and postoperative AL values in the same patient. In the ERV subgroup, Bland-Altman plots were used to examine the addition and proportional error between the pre- and postoperative AL values measured using the OCTB1-ERV mode. A $p$-value $<0.05$ was considered statistically significant.

\section{Results}

\section{Comparison of AL Acquisition Rate between Two} OCT Biometers

In all the 1,969 eyes, the acquisition rate of the AL measurement was $94.1 \%$ ( 1,853 eyes) and $95.6 \%$ ( 1,882 eyes) by OCTB2 and OCTB1 standard mode, respectively. The acquisition rate in OCTB1 was significantly higher than OCTB2 ( $p=0.037$, adjusted residual was 2.1$)$. Furthermore, in the OCTB1-ERV mode, 64 eyes (73.7\%) were successfully measured out of 87 eyes that failed to be measured in the standard mode. A total of 1,946 eyes (98.8\%) were measured as AL by OCTB1. The AL acquisition rate of OCTB1, including in the ERV mode, was significantly higher than that of OCTB2 $(p<0.00001$; adjusted residual was 8.0).

In 213 eyes of grade 4 or higher, acquisition rate of $\mathrm{AL}$ for OCTB1-standard mode (148 eyes, 69.5\%) was not significantly different from that for OCTB2 (131 eyes, 61.5\%) $(p=0.083)$. However, the overall acquisition rate for OCTB1 including standard and ERV modes (199 eyes, 93.4\%) was significantly higher than that for OCTB2 ( $p<$ 0.001; Fig. 2a. Among eyes for which AL could not be measured using the OCTB1-standard mode, that of one eye $(1.5 \%)$ could be measured using OCTB2. In contrast, among eyes for which AL could not be measured using OCTB2, that of 18 eyes (22.0\%) could be measured using the OCTB1-standard mode. Among the cases in which AL could not be measured with the OCTB1-standard mode (65 eyes), 51 eyes (78.5\%) were successfully measured with the OCTB1-ERV mode (ERV subgroup). Of the 213 eyes with a grade 4 or higher, 14 eyes (6.6\%) could not be measured in the ERV mode. Among the 82 eyes that could not be measured using OCTB2, 25 eyes (30.5\%) presented white cataract; of these 25 , AL of 4 eyes (16.0\%) could be measured using the OCTB1-standard mode, and AL of 15 eyes $(60.0 \%)$ could be measured using the ERV mode. 
Fig. 3. Comparison of AL measurement accuracy between OCTB1 with the standard mode, OCTB1 with the ERV mode, OCTB2, and US biometer. AL measurement accuracy was evaluated by comparing pre- and postoperative AL values. The absolute difference of AL value measured using OCTB1 with the standard mode was significantly smaller than that measured using OCTB1 with the ERV mode ( $p=$ $0.008)$, OCTB2 $(p<0.001)$, and US biometer $(p<0.001)$. AL, axial length; US, ultrasound; ERV, enhanced retina visualization; OCTB1, optical coherence tomography biometer using the ARGOS device; OCTB2, optical coherence tomography biometer using the IOLMaster700 device.

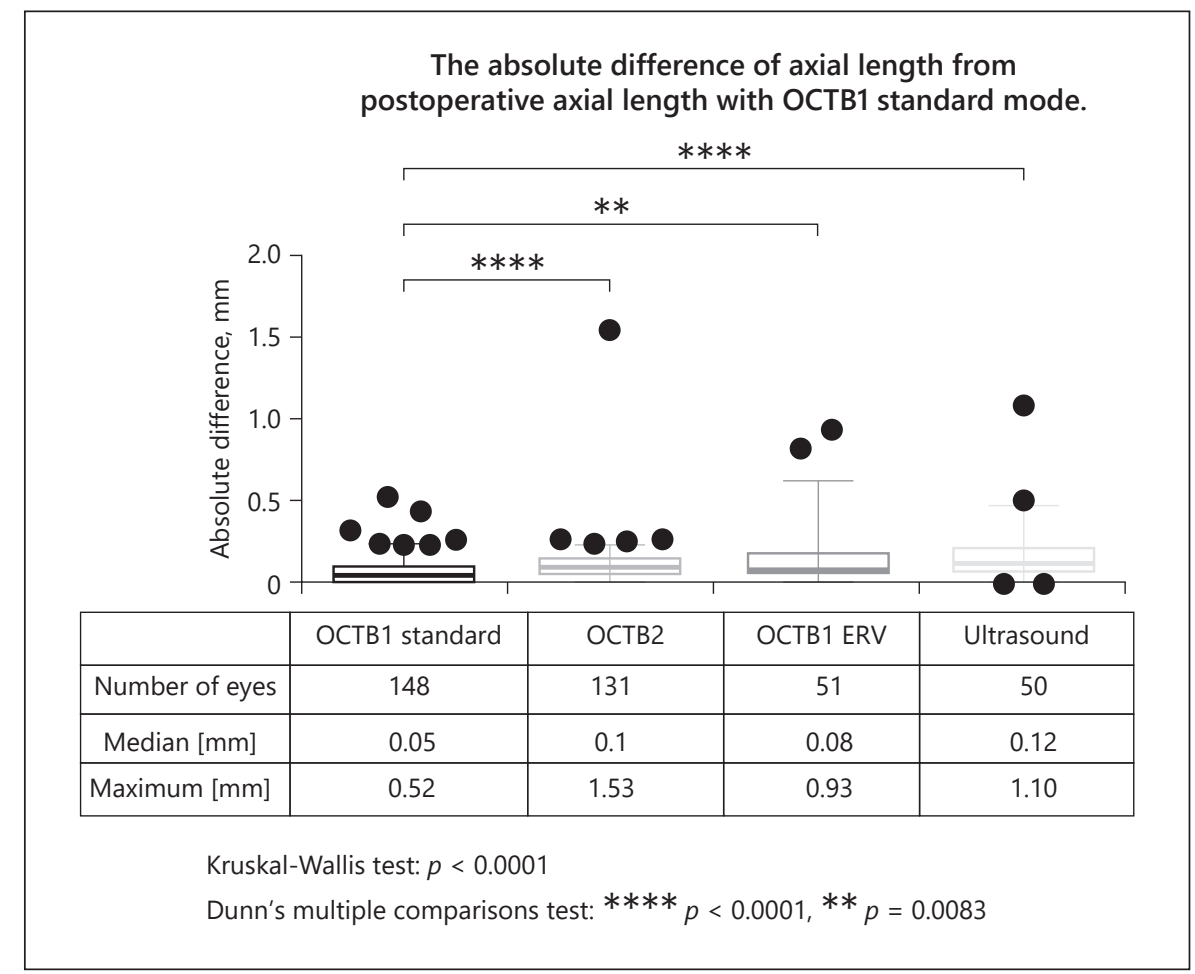

\section{Accuracy of AL Measurement Using ERV Mode in} Dense Cataract

The differences between pre- and postoperative AL values were evaluated in the ERV subgroup. The preoperative AL was significantly longer than the postoperative AL in the ERV subgroup ( $p=0.004$; median of AL difference, $0.055 \mathrm{~mm}$ ). The correlation coefficient between pre- and postoperative AL was 0.993 , and the $95 \%$ confidence interval was $0.0-0.110 \mathrm{~mm}$. In the ERV subgroup, the difference between pre- and postoperative AL was $\leq 0.2 \mathrm{~mm}$ in 40 eyes $(78.4 \%)$ and $\leq 0.1 \mathrm{~mm}$ in 30 eyes (58.8\%; Fig. 2b). In 17 eyes with white cataract, the mean absolute difference was $0.13 \pm 0.11 \mathrm{~mm}(0.0-0.36 \mathrm{~mm})$.

\section{Comparison of AL Measurement Accuracy among}

OCTB1 with the Standard Mode, OCTB2, OCTB1

with the ERV Mode, and US Biometer

Median absolute differences between pre- and postoperative AL values for OCTB1-standard mode, OCTB2, OCTB1-ERV mode, and US biometer were 0.05, 0.10, 0.08 , and $0.12 \mathrm{~mm}$, respectively. The absolute difference for the OCTB1-standard mode was significantly lower than that for OCTB2, OCTB1-ERV mode, and US biometer $(p<0.001, p=0.008$, and $p<0.001$, respectively; Fig. 3).
Agreement Analyses for Pre- and Postoperative AL Measurement Using OCB1 with ERV Mode

Agreement of AL value measured using OCB1 with the ERV mode was analyzed using Bland-Altman plots (Fig. 4). There were neither additional nor proportional errors between the pre- and postoperative AL values measured using the OCTB1-ERV mode. According to the patient chart review, the two outliers (Fig. 4, arrow) were found to be patients with unstable eye fixation during examination owing to amblyopia or posterior staphyloma.

\section{Discussion}

Previous studies have reported that the SS-OCT biometer has a higher $\mathrm{AL}$ acquisition rate than does PCI [14, $22,23]$. In two of these studies, it was reported that the AL acquisition rate of the SS-OCT biometer was 87.5 and $91.3 \%$, respectively, in eyes with advanced cataract, in which measurements could not be made using PCI (IOLMaster500) $[14,23]$. The ERV mode is a method for measuring the AL by applying enhanced depth imaging technology, but its clinical efficacy is not known. In this study, we evaluated the acquisition rate and accuracy of AL mea- 
Fig. 4. Bland-Altman plot: analysis of agreement between pre- and postoperative AL values measured using OCTB1 with the ERV mode. There were no additions or proportional errors between the two measurements. Black arrows show outliers. 95\% confidence interval: $0.007-0.1323$ $\mathrm{mm}, 95 \%$ limit of agreement: $-0.367-0.506$ $\mathrm{mm}$. ERV, enhanced retina visualization; $\mathrm{AL}$, axial length; OCTB1, optical coherence tomography biometer using the ARGO device.

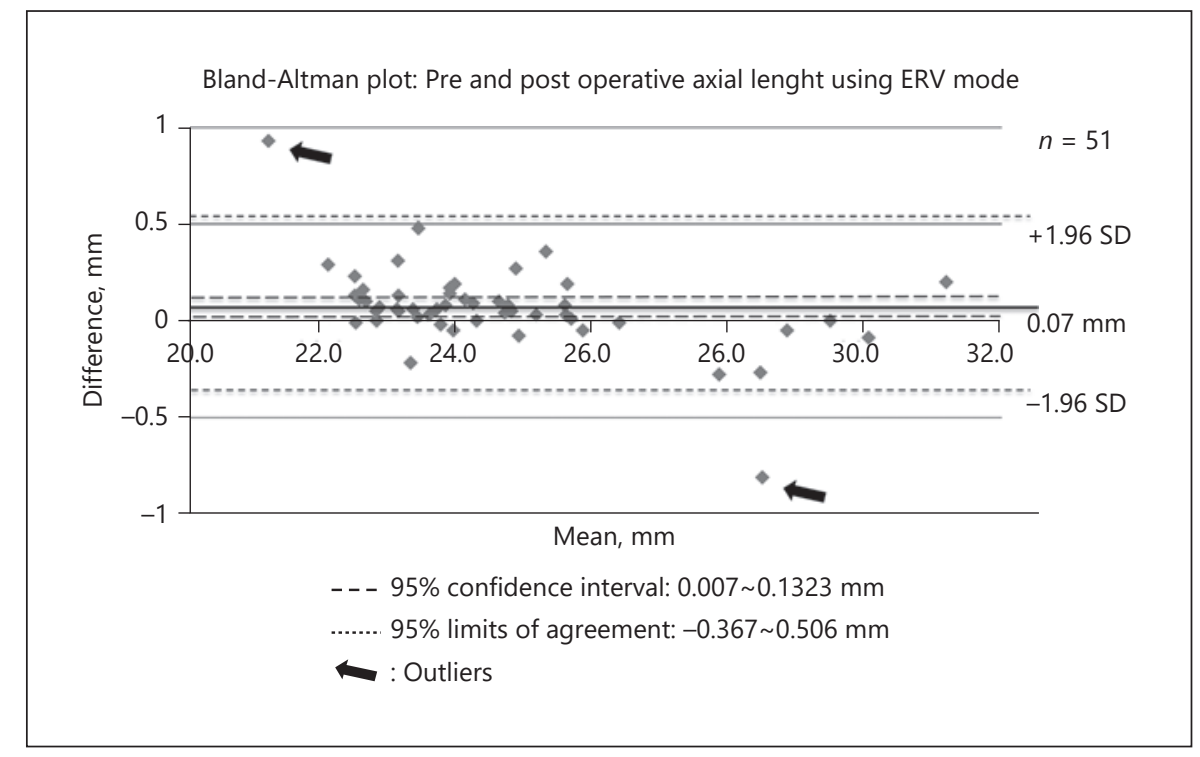

surement using the ERV mode when the standard mode of OCTB1 could not be measured.

In this study, the acquisition rate of AL in all cases was significantly higher in the standard mode of OCTB1 $(95.6 \%)$ than in OCTB2 (94.1\%). However, judging from these values, the clinical difference was small. However, the AL acquisition rate of OCTB1 - including the ERV mode - was $98.8 \%$, which is considered clinically higher than that of OCTB2.

In this study, for dense cataract with a nuclear hardness grade of $\geq 4$ or for white cataract, the $\mathrm{AL}$ acquisition rates using the OCTB1-standard mode and OCTB 2 were 69.5 and $61.5 \%$, respectively. These numbers were lower than those reported for a dense cataract by previous studies $[12,23]$. Our hospital accepts many patients with advanced cataract from wide geographical regions in Japan. Among them, patients in whom AL measurement had already failed in the former ophthalmology clinic were included. In addition, the nucleus grade of cataract was classified by using the Emery-Little classification system in this study; however, the definition of advanced cataract differs depending on the study. As a result, the grade of cataract among patients included in each study varies, which may have a large influence on the acquisition rate. Therefore, it is difficult to directly compare the AL acquisition rate with those reported by previous studies.

$\mathrm{AL}$ of a total of $93.4 \%$ of dense cataract cases was successfully measured using OCTB1. The AL acquisition rate of OCTB1-standard mode was $69.5 \%$, but the AL of the remaining $23.9 \%$ was measurable in the ERV mode.
As a result, the AL acquisition rate of OCTB1 was significantly higher than that of OCTB2, which was consistent with the result published in a previous report [18]. In general, because white cataract significantly impairs the passage of light owing to light scattering, it is difficult to measure AL in such cases even using the SS-OCT biometer with long-wavelength light. Considering that AL of $48.6 \%$ of white cataract cases that could not be measured using the OCTB1-standard mode was successfully measured using the OCTB1-ERV mode, the usefulness of ERV mode seems to be higher. However, patients with mature cataracts, including white cataracts, tend to have poor visual acuity, and reliable foveal fixation is difficult. Therefore, we should be careful when using the measurement results for intraocular lens power calculation.

Regarding the comparison of pre- and postoperative ALs, the median of the absolute error in OCTB1-ERV mode was $0.08 \mathrm{~mm}$, which was larger than that in the OCTB1-standard mode. Generally, in eyes with standard $\mathrm{AL}$, a measurement error of $0.1 \mathrm{~mm}$ in the AL may cause a measurement error of about $0.27-0.3 \mathrm{D}[3,4,24]$. Therefore, the error that occurs with the use of OCTB1-ERV mode seems to be clinically negligible. Moreover, considering that the absolute error of US biometer in the same patient group was $0.12 \mathrm{~mm}$, the error that occurred with the use of the ERV mode was considered to be almost equivalent to that of US biometer; therefore, ERV mode was considered clinically useful. However, even with the use of the ERV mode, there were eyes in which AL could not be measured, and $22 \%$ of the cases showed an AL mea- 
surement error of $\geq 0.2 \mathrm{~mm}$. It is important to measure $\mathrm{AL}$ using the US biometer and to check if there is a large difference even if the AL was measured using the ERV mode.

In this study, we observed a large error in two cases in which AL was measured using the ERV mode. One was an eye with amblyopia, and the other was an eye with staphyloma. In both cases, eye fixation to the target was difficult, and it was considered that the biometer could not accurately capture fovea because of the combined effects with advanced cataract.

The Bland-Altman plot analysis was performed to investigate the characteristics of dense cataract that requires the ERV mode. According to the Bland-Altman plot anal$y$ sis, there was no addition or proportional error in the $\mathrm{AL}$ measurement before and after cataract surgery using the ERV mode. The error in the ERV mode may be caused by the deviation from the visual axis owing to the poor eye fixation. OCTB1 measures AL using the segmental refractive indices. However, a uniform value of the refractive index of the crystalline lens is used regardless of the degree of cataract. Especially, the difference in the refractive index of the crystalline lens in cases with a dense cataract may have a greater effect on AL measurement than that in those with mild to moderate cataracts. In the future, if it is possible to individually adjust and calculate the refractive index depending on the degree of cataract, the accuracy may be further improved.

In our facility, when a patient with a low visual acuity could not confirm the fixation target, the examiner guided the eye direction while looking at the live whole eye OCT image displayed on the OCTB1 measurement screen. Therefore, it seems that even in patients with poor visual acuity, the practical AL could be measured using the ERV mode in many cases. When measuring the AL of patients with poor visual acuity using OCTB1, the usage of whole eye OCT image may be important to guide sufficient eye fixation.

This study had several limitations. First, the number of examiners was limited because it was a single-center retrospective study. Especially when measuring in ERV mode, it is important to guide the patient's eye fixation accurately, and hence, the experience of the examiner may influence the accuracy of AL measurement in the ERV mode. In the future, it is necessary to examine the results used by many examiners at multiple facilities. Second, in this study, only the nuclear hardness was graded by the Emery-Little classification system. However, as the strong turbidity on the visual axis - such as both the anterior and the posterior subcapsular cataract - affects the AL acquisition rate, using other classification systems - such as the
LOCS III - could tamper the results of this study. Further studies should be conducted using a more detailed cataract classification system, such as the LOCSIII, in the future. Third, in this study, because the ERV mode was used only in cases where the AL could not be measured in the standard mode, it was not possible to evaluate the accuracy of ERV mode in cases where AL were measured in standard mode. In the future, a prospective study should be done to evaluate the accuracy of the ERV mode in cases in which the standard mode can be measured. Fourth, multiple intraocular lenses were used in this study. There were 8 types of acrylic IOL and one type of silicone IOL implanted in this study population. We examined the accuracy of the ERV mode by comparing pre- and postoperative AL measurements. In OCTB1, the segmental refractive index is used for AL measurement. In this study, for all acrylic IOLs, the same refractive index (1.550) was applied. However, it might be different in different types of acrylic IOL. In the future, studies using a single type of IOL will be necessary. Fifth, the study evaluated the accuracy of AL measurement using the ERV mode of OCTB1. Many of the patients who were included in this study have ocular diseases other than cataracts, and postoperative refractive error could not be evaluated in all cases. Further studies evaluating the axial length and the final predictive refractive error should be considered in a larger number of cases. In conclusion, the current study showed that the ERV mode could be a promising function to measure AL especially in patients with advanced cataracts including white cataracts.

\section{Acknowledgement}

This article does not include any nonauthor contributors to acknowledge.

\section{Statement of Ethics}

This retrospective study was approved by the Institutional Review Board of Japan Community Health Care Organization Chukyo Hospital (\#2017026). The protocol of this study followed the tenets of the Declaration of Helsinki. The opt-out method was approved by the institutional review board in place of obtaining informed consent.

\section{Conflict of Interest Statement}

Dr. Tamaoki reports receiving personal fees from Nidek and Alcon Japan outside the submitted work. Dr. Kojima reports receiving personal fees from STAAR Surgical, Santen Pharmaceuti- 
cal Co. Ltd., Otsuka Pharmaceutical, Johnson \& Johnson, and Alcon Japan outside of the submitted work. Dr. Hasegawa and Ms. Yamamoto have no commercial interests to disclose. Dr. Kaga reports receiving personal fees from Santen Pharmaceutical and Alcon Japan outside the submitted work. Dr. Tanaka has no commercial interests to disclose. Dr. Ichikawa reports receiving personal fees from Santen Pharmaceutical Co. Ltd., Alcon Japan, Kowa, and Carl Zeiss Meditec outside the submitted work. In addition, Dr. Ichikawa has two patents (2012-005573 and 2011218076), both licensed to Kazuo Ichikawa.

\section{Funding Sources}

We did not receive any funding relevant to this study.

\section{Author Contributions}

All authors met the four conditions shown in the ICMJE Criteria for Authorship. Conception or design of the work: Tamaoki A., Kojima T., Kaga T., Tanaka K., and Ichikawa K. Data collection: Tamaoki A., Hasegawa A., Yamamoto M., and Kaga T. Data analysis and interpretation: Tamaoki A., Kojima T., Hasegawa A., Yamamoto M., Kaga T., Tanaka K., and Ichikawa K. Drafting of the article: Tamaoki A. and Kojima T. Critical revision of the article: Hasegawa A., Yamamoto M., Kaga T., Tanaka K., and Ichikawa K. Final approval of the version to be published: Tamaoki A., Kojima T., Hasegawa A., Yamamoto M., Kaga T., Tanaka K., and Ichikawa $\mathrm{K}$. The current study was presented as a poster at the annual meeting of European Society of Cataract and Refractive Surgery on September 30, 2019, in Paris, France. Partial results of this study were also presented in the 2018 annual meeting of Association for Research in Vision and Ophthalmology on May 1 in Hawaii, USA.

\section{References}

1 Kershner RM. Refractive cataract surgery. Curr Opin Ophthalmol. 1998 Feb;9(1):46-54.

2 Olsen T. Sources of error in intraocular lens power calculation. J Cataract Refract Surg. 1992 Mar;18(2):125-9.

3 Olsen T. Calculation of intraocular lens power: a review. Acta Ophthalmol Scand. 2007 Aug;85(5):472-85.

4 Norrby S. Sources of error in intraocular lens power calculation. J Cataract Refract Surg. 2008 Mar;34(3):368-76.

5 Findl O, Kriechbaum K, Sacu S, Kiss B, Polak $\mathrm{K}, \mathrm{Nepp}$ J, et al. Influence of operator experience on the performance of ultrasound biometry compared to optical biometry before cataract surgery. J Cataract Refract Surg. 2003 Oct;29(10):1950-5.

6 Findl O, Drexler W, Menapace R, Hitzenberger CK, Fercher AF. High precision biometry of pseudophakic eyes using partial coherence interferometry. J Cataract Refract Surg. 1998 Aug;24(8):1087-93.

7 Haigis W, Lege B, Miller N, Schneider B. Comparison of immersion ultrasound biometry and partial coherence interferometry for intraocular lens calculation according to Haigis. Graefes Arch Clin Exp Ophthalmol. 2000 Sep;238(9):765-73.

8 Verhulst E, Vrijghem JC. Accuracy of intraocular lens power calculations using the Zeiss IOL master. A prospective study. Bull Soc Belge Ophtalmol. 2001;281(281):61-5.

9 Connors R3rd, Boseman P3rd, Olson RJ. Accuracy and reproducibility of biometry using partial coherence interferometry. J Cataract Refract Surg. 2002 Feb;28(2):235-8.

10 Kiss B, Findl O, Menapace R, Wirtitsch M, Petternel V, Drexler W, et al. Refractive out- come of cataract surgery using partial coherence interferometry and ultrasound biometry: clinical feasibility study of a commercial prototype II. J Cataract Refract Surg. 2002 Feb;28(2):230-4.

11 Freeman G, Pesudovs K. The impact of cataract severity on measurement acquisition with the IOLMaster. Acta Ophthalmol Scand. 2005 Aug;83(4):439-42.

12 McAlinden C, Wang Q, Pesudovs K, Yang X, Bao F, Yu A, et al. Axial length measurement failure rates with the IOLMaster and Lenstar LS 900 in eyes with cataract. PLoS One. 2015 Jun;10(6):e0128929.

13 McAlinden C, Wang Q, Gao R, Zhao W, Yu A, Li Y, et al. Axial length measurement failure rates with biometers using swept-source optical coherence tomography compared to partial-coherence interferometry and optical low-coherence interferometry. Am J Ophthalmol. 2017 Jan;173:64-9.

14 Hirnschall N, Varsits R, Doeller B, Findl O. Enhanced penetration for axial length measurement of eyes with dense cataracts using swept source optical coherence tomography: a consecutive observational study. Ophthalmol Ther. 2018 Jun;7(1):119-24.

15 Tehrani M, Krummenauer F, Blom E, Dick HB. Evaluation of the practicality of optical biometry and applanation ultrasound in 253 eyes. J Cataract Refract Surg. 2003 Apr;29(4): 741-6.

16 van den Berg TJ, Coppens JC. Conversion of lens slit lamp photographs into physical lightscattering units. Invest Ophthalmol Vis Sci. 1999 Aug;40(9):2151-7.

17 Huang J, Chen H, Li Y, Chen Z, Gao R, Yu J, et al. Comprehensive comparison of axial length measurement with three swept-source oct-based biometers and partial coherence interferometry. J Refract Surg. 2019 Feb 1;35(2): 115-20.

18 Tamaoki A, Kojima T, Hasegawa A, Yamamoto M, Kaga T, Tanaka K, et al. Clinical evaluation of a new swept-source optical coherence biometer that uses individual refractive indices to measure axial length in cataract patients. Ophthalmic Res. 2019;62(1):11-23.

19 Ademola-Popoola DS, Nzeh DA, Saka SE, Olokoba LB, Obajolowo TS. Comparison of ocular biometry measurements by applanation and immersion A-scan techniques. J Curr Ophthalmol. 2015 Sep-Dec;27(3-4):110-4.

20 Wong IY, Koizumi H, Lai WW. Enhanced depth imaging optical coherence tomography. Ophthalmic Surg Lasers Imaging. 2011 Jul;42(Suppl):S75-84.

21 Emery JM, Little JH. Phacoemulsification and aspiration of cataract: surgical techniques, complications, and results. St Louis: CV Mosby; 1979.

22 Akman A, Asena L, Güngör SG. Evaluation and comparison of the new swept source OCT-based IOLMaster 700 with the IOLMaster 500. Br J Ophthalmol. 2016 Sep;100(9): 1201-5.

23 Chirapapaisan C, Srivannaboon S, Chonpimai P. Efficacy of swept-source optical coherence tomography in axial length measurement for advanced cataract patients. Optom Vis Sci. 2020 Mar;97(3):186-91.

24 Shammas HJ, Wetterwald N, Potvin R. New mode for measuring axial length with an optical low-coherence reflectometer in eyes with dense cataract. J Cataract Refract Surg. 2015 Jul;41(7):1365-9.
Axial Length Measurement Using

Enhanced Retina Visualization Mode
Ophthalmic Res 2021;64:595-603

DOI: $10.1159 / 000515054$ 